\title{
Noncommutative Geometry Spectral Action as a framework for unification: Introduction and phenomenological/cosmological consequences
}

\author{
Mairi Sakellariadou* \\ Department of Physics, King's College, University of London, Strand WC2R 2LS, London, U.K.
}

\begin{abstract}
I will summarize Noncommutative Geometry Spectral Action, an elegant geometrical model valid at unification scale, which offers a purely gravitational explanation of the Standard Model, the most successful phenomenological model of particle physics. Noncommutative geometry states that close to the Planck energy scale, space-time has a fine structure and proposes that it is given as the product of a four-dimensional continuum compact Riemaniann manifold by a tiny discrete finite noncommutative space. The spectral action principle, a universal action functional on spectral triples which depends only on the spectrum of the Dirac operator, applied to this almost commutative product geometry, leads to the full Standard Model, including neutrino mixing which has Majorana mass terms and a see-saw mechanism, minimally coupled to gravity. It also makes various predictions at unification scale. I will review some of the phenomenological and cosmological consequences of this beautiful and purely geometrical approach to unification.
\end{abstract}

PACS numbers: 11.10.Nx, 04.50.+h, 12.10.-g, 11.15.-q, 12.10.Dm

\section{PREFACE}

It is with great pleasure that I am writing this contribution for Mario Castagnino's Festschrift. I have met Mario at the beginning of my career, almost twenty years ago, and I have decided to write here on a subject which captured my interest only very recently. In what follows, I will summarize NonCommutative Geometry Spectral Action, and then discuss shortly some of its phenomenological and cosmological consequences. Since Mario has worked in various mathematical as well as physical problems, I hope that my choice for this contribution is the right one.

\section{INTRODUCTION}

One of the fundamental questions in theoretical physics is the origin of space-time, an issue which is closely related to unifying all fundamental interactions including gravity, and as such is not independent of solving the issue of Quantum Gravity. The motivation of studying NonCommutative Geometry (NCG) is at least two-fold. Namely, besides the mathematical beauty of the NCG theory, this approach leads to a variety of cosmological and high energy physics consequences. Let me be a bit more precise. As far as Cosmology is concerned, early universe cosmological models can be tested with very accurate astrophysical data, while high energy experiments, and in particular the Large Hadron Collider (LHC), will test some of the theoretical pillars of these models. However, despite the golden era of cosmology, issues like the origin of dark energy, the hunting for the successful dark

\footnotetext{
*mairi.sakellariadou@kcl.ac.uk
}

matter candidate, or the search for a natural and wellmotivated inflationary model, are still awaiting for a definite answer. The main approaches upon which cosmological models have been built is either String Theory or Loop Quantum Gravity ${ }^{1}$. Here, we will use NCG Spectral Action. As High Energy Physics is concerned, one may argue that the distinct feature between the diffeomorphism invariance (outer automorphism) which governs General Relativity, and the local gauge invariance (inner automorphism) which governs gauge symmetries, may be at the origin of the unsuccessful search for a unified theory of all interactions including gravity. Moreover, there is a whole list of questions within the realm of particle physics, awaiting for a definite answer from the successful unified theory of all interactions. In particular, there is no conceptual justification for the choice of the gauge group $\mathrm{G}_{\mathrm{SM}}=\mathrm{SU}(3) \times \mathrm{SU}(2) \times \mathrm{U}(1)$ of the Standard Model (SM) of electroweak and strong interactions, nor is any reason for the various representations for the fermions and bosons in the construction of the SM.

Following the spirit of NCG [1, 2], one may ague that much below Planck scale, gravity can be safely considered as a classical theory, while as energies approach Planck scale, the quantum nature of space-time reveals itself, and the naive approach, valid however at low energy scales, that physics can be described by the sum of the Einstein-Hilbert and the SM action becomes just an invalid approximation. To guess the appropriate structure of space-time at Planckian energy scales is a rather dangerous issue, and one is often making an extrapolation by (too) many orders of magnitude. In this sense, NCG

\footnotetext{
${ }^{1}$ Cosmological models have been also, to a less extent, built upon other variants of Quantum Gravity, like Spin Foams, WheelerDe Witt equation, Causal Dynamical Triangulations, or Causal Sets.
} 
Spectral Action follows a rather safer approach, namely it looks for a hidden structure in the functional of gravity coupled to the SM at today's (low) energy scales.

In what follows, we will adopt an effective theory approach and consider the simplest case beyond commutative spaces, namely we will suppose that at energies below but close to the Planck scale, space-time is almost commutative. At higher energy scales, space-time should become noncommutative in a nontrivial way, while at energies above the Planck scale the whole concept of geometry may altogether become meaningless.

\section{NCG SPECTRAL ACTION}

The basic idea of the NCG Spectral Action approach, based upon three ansatz, is that the Standard Model of electroweak and strong interactions should be seen as a phenomenological model, which however dictates the appropriate geometry of space-time, so that the MaxwellDirac action functional leads to the SM action. This proposal then implies that the geometrical space should be given by the tensor product $\mathcal{M} \times \mathcal{F}$ of a continuum compact ${ }^{2}$ Riemannian manifold $\mathcal{M}$ (geometry for spacetime) and a tiny discrete finite noncommutative space $\mathcal{F}$ (internal geometry for the SM) composed of just two points. The finite geometry $\mathcal{F}$ will be chosen so that it is one of the simplest and most natural finite noncommutative geometries of the right dimension to solve the fermion doubling problem. The choice of $\mathcal{M} \times \mathcal{F}$ as the appropriate geometrical space consists the first ansatz.

The noncommutative nature of the discrete space $\mathcal{F}$ is given by a spectral triple ${ }^{3}(\mathcal{A}, \mathcal{H}, \mathcal{D})$, where $\mathcal{A}$ is an involution of operators on the finite-dimensional Hilbert space $\mathcal{H}$ of Euclidean fermions, and $\mathcal{D}$ is a self-adjoint unbounded operator in $\mathcal{H}$, such that $J \mathcal{D}=\epsilon^{\prime} \mathcal{D} J$, where $J$ is an antilinear isometry of the finite dimensional Hilbert space $\mathcal{H}, J: \mathcal{H} \rightarrow \mathcal{H}$, with the properties

$$
J^{2}=\epsilon, \quad J \gamma=\epsilon^{\prime \prime} \gamma J,
$$

where $\gamma$ is the chirality operator and $\epsilon, \epsilon^{\prime}, \epsilon^{\prime \prime} \in\{ \pm 1\}^{3}$. Following the spirit of NCG spectral action, the SM minimally coupled with Einstein gravity appears [3] naturally as pure gravity on the $\mathcal{M} \times \mathcal{F}$ space.

Let me first discuss the distinction between the metric (or spectral) dimension, given by the behavior of the eigenvalues of the Dirac operator, and the K-theoretic

\footnotetext{
2 The Euclidean space-time manifold is taken to be compact for simplicity.

3 Applying the definition of spectral type in the context of noncommutative geometry, it must be seen as setting a unitary Hilbert space representation of a setup that allows to manipulate algebraically coordinates and measure distances.
}

dimension, an algebraic dimension based on K-theory. I will start with the metric dimension: Since the relevant Dirac operator for space-time is the ordinary Dirac operator on a curved space-time, the metric dimension is equal to four. The internal Dirac operator consists of the fermionic mass matrix, which has a finite number of eigenvalues, and therefore the internal metric dimension is equal to zero. As a result, the metric dimension of the $\mathcal{M} \times \mathcal{F}$ geometry is just four, the same as that of the ordinary space-time manifold. I will now discuss the K-theoretic dimension: There are 8 possible combinations for $\epsilon, \epsilon^{\prime}, \epsilon^{\prime \prime}$ and this defines a K-theoretic dimension of noncommutative space modulo 8 . To resolve the fermion doubling problem, by projecting out the unphysical degrees of freedom resting in the internal space, the real structure of the finite geometry $\mathcal{F}$ turns out to be such that its K-theoretic dimension is equal to six [4, 5], and in this case $\left(\epsilon, \epsilon^{\prime}, \epsilon^{\prime \prime}\right)=(1,1,-1)$. Thus, the Ktheoretic dimension of the product space $\mathcal{M} \times \mathcal{F}$ is equal to $10 \sim 2$ modulo 8 , allowing one to impose simultaneously the reality and Weyl conditions in the Minkowskian continued forms.

It becomes then clear that the reason for introducing $\mathcal{F}$ is to correct the $\mathrm{K}$-theoretic dimension from four to ten (modulo 8). In other words, the fermion doubling problem requires [4, 5] crossing the ordinary four-dimensional continuum by a space of K-theoretic dimension 6 . Classifying all irreducible finite noncommutative geometries of K-theoretic dimension six, it was shown [6] that the dimension (per generation) is a square of an integer $k$.

Let us now go back to the discussion about the spectral triple $(\mathcal{A}, \mathcal{H}, \mathcal{D})$. The algebra $\mathcal{A}$, related to the gauge group of local gauge transformations, is the algebra of coordinates. Within NCG, all information about a space is encoded in the algebra of coordinates $\mathcal{A}$. By assuming that the algebra constructed in the product geometry $\mathcal{M} \times \mathcal{F}$ is symplectic-unitary, $\mathcal{A}$ must be of the form [7]

$$
\mathcal{A}=M_{a}(\mathbb{H}) \oplus M_{k}(\mathbb{C}),
$$

with $k=2 a$ and $\mathbb{H}$ being the algebra of quaternions. The field of quaternions $\mathbb{H}$ plays an important rôle in this construction and its choice remains to be explained. To obtain the SM we will assume quaternion linearity.

The first possible value for the even number $k$ is 2 , corresponding to a Hilbert space of four fermions; it is ruled out from the existence of quarks. The second one, $k=4$, leads to the correct number of $k^{2}=16$ fermions in each of the three generations. Certainly if at LHC new particles are discovered, one may be able to accommodate them by including a higher value for the even number $k$. Notice that considering three generations $(N=3)$ is a physical input in NCG. Certainly, as far as physics is concerned, violation of $\mathrm{CP}$ is a reason for setting $N \geq 3$, but one would like to come up with also a mathematical justification. The choice of $\mathcal{A}$ is the main input in the 
entire approach and its choice consists the second ansatz.

The choice of Hilbert space has no importance, since all separable infinite-dimensional Hilbert spaces are isomorphic.

The operator $D$ corresponds to the inverse of the Euclidean propagator of fermions, and is given by the Yukawa coupling matrix which encodes the masses of the elementary fermions and the Kobayashi-Maskawa mixing parameters. The commutator $[D, a]$, with $a \in \mathcal{A}$, plays the rôle of the differential quotient $d a / d s$, with $d s$ the unit of length. The familiar geodesic formula

$$
d(x, y)=\inf \int_{\gamma} d s
$$

where the infimum is taken over all possible paths connecting $x$ to $y$, which is used to determine the distance $d(x, y)$ between two points $x$ and $y$ within Riemannian geometry, is replaced by

$$
d(x, y)=\sup \{|f(x)-f(y)|: f \in \mathcal{A},\|[D, f]\| \leq 1\},
$$

where $D$ is the inverse of the line element $d s$, within the noncommutative spectral geometry.

The operator $D$ is assumed [3] to commute with the sub-algebra $(\lambda, \lambda, 0) ; \lambda \in \mathbb{C}$. The meaning of this condition is clear as far as physics is concerned, namely it means that the photon is massless, however a conceptual mathematical reason for only considering metrics satisfying this requirement is lacking.

The fermions of the SM provide the Hilbert space $\mathcal{H}$ of a spectral triple for the algebra $\mathcal{A}$, while the bosons of the SM, including the Higgs boson, are obtained through inner fluctuations of the Dirac operator of the product $\mathcal{M} \times \mathcal{F}$ geometry. Hence, the Higgs boson, which generates the masses of elementary particles through Spontaneous Symmetry Breaking, becomes just a gauge field corresponding to a finite difference. Note that the corresponding mass scale specifies the inverse size of the discrete geometry $\mathcal{F}$.

To obtain the full Lagrangian of the SM, minimally coupled to gravity, we will apply the Spectral Action principle, which consists the third ansatz. It states that the Dirac operator connects the two pieces of the product $\mathcal{M} \times \mathcal{F}$ geometry nontrivially. The spectral action functional ${ }^{4} S$ depends only of the spectrum of the Dirac operator and is of the form $\operatorname{Tr}(f(D / \Lambda))$, with $\Lambda$ giving the energy scale ${ }^{5}$ and $f$ being a cut-off function, whose choice plays only a small rôle. Note that both $D$ and

\footnotetext{
${ }^{4}$ Similar to Fourier Transform in commutative geometry.

5 The arbitrary mass scale in the spectral action for the Dirac operator can be made dynamical by introducing a dilaton field, which guarantees the scale invariance of the SM interactions, and provides a mechanism to generate mass hierarchies [8].
}

$\Lambda$ have physical dimensions of a mass and there is no absolute scale on which they can be measured.

Using heat kernel methods the trace $\operatorname{Tr}(f(D / \Lambda))$ can be written in terms of the geometrical Seeley-deWitt coefficients $a_{n}$, as [9]

$$
\sum_{n=0}^{\infty} F_{4-n} \Lambda^{4-n} a_{n}
$$

where the function $F$ is defined such that $F\left(D^{2}\right)=f(D)$. Defining the moments

$$
f_{k} \equiv \int_{0}^{\infty} f(u) u^{k-1} \mathrm{~d} u \quad, \text { for } k>0
$$

and $f_{0} \equiv f(0)$, one finds

$$
\begin{aligned}
F_{4} & =2 f_{4}, \\
F_{2} & =2 f_{2}, \\
F_{0} & =f_{0}, \\
F_{-2 n} & =\left[(-1)^{n}\left(\frac{\mathrm{d}}{2 u \mathrm{~d} u}\right)^{n} f\right](0) \text { for } n \geq 1,
\end{aligned}
$$

while the Seeley-deWitt coefficients $a_{n}$ are known for any second order elliptic differential operator.

The spectral action can be expanded in powers of the scale $\Lambda$ in the form [3, 10, 11]

$\operatorname{Tr}\left(f\left(\frac{D}{\Lambda}\right)\right) \sim \sum_{k \in \text { DimSp }} f_{k} \Lambda^{k} f|D|^{-k}+f(0) \zeta_{D}(0)+\mathcal{O}(1)$,

where $f_{k}$ are the momenta of the function $f$ given in Eq. (6), the noncommutative integration is defined in terms of residues of zeta functions, $\zeta_{D}(s)=\operatorname{Tr}\left(|D|^{-s}\right)$ at poles of the zeta function, and the sum is over points in the dimension spectrum of the spectral triple.

Considering the Riemannian geometry to be fourdimensional, the asymptotic expansion of the trace reads [12]

$$
\begin{array}{r}
\operatorname{Tr}\left(f\left(\frac{D}{\Lambda}\right)\right) \sim 2 \Lambda^{4} f_{4} a_{0}+2 \Lambda^{2} f_{2} a_{2}+f_{0} a_{4}+\cdots \\
+\Lambda^{-2 k} f_{-2 k} a_{4+2 k}+\cdots .
\end{array}
$$

The smooth even function $f$, which decays fast at infinity, only enters in the multiplicative factors:

$$
\begin{aligned}
f_{4} & =\int_{0}^{\infty} f(u) u^{3} d u, \\
f_{2} & =\int_{0}^{\infty} f(u) u d u, \\
f_{0} & =f(0), \\
f_{-2 k} & =(-1)^{k} \frac{k !}{(2 k) !} f^{(2 k)}(0) .
\end{aligned}
$$


Since $f$ is taken as a cut-off function, its Taylor expansion at zero vanishes, thus its asymptotic expansion, Eq. (9), reduces to just

$$
\operatorname{Tr}\left(f\left(\frac{D}{\Lambda}\right)\right) \sim 2 \Lambda^{4} f_{4} a_{0}+2 \Lambda^{2} f_{2} a_{2}+f_{0} a_{4} .
$$

Hence, $f$ plays a rôle through only its momenta $f_{0}, f_{2}, f_{4}$, which are three additional real parameters in the model, physically related to the coupling constants at unification, the gravitational constant, and the cosmological constant. In this four-dimensional case, the term in $\Lambda^{4}$ gives a cosmological term, the term in $\Lambda^{2}$ gives the Einstein-Hilbert action functional with the physical sign for the Euclidean functional integral (provided $f_{2}>0$ ), and the $\Lambda$-independent term yields the Yang-Mills action for the gauge fields corresponding to the internal degrees of freedom of the metric. The scale-independent terms in the spectral action have conformal invariance.

The spectral action functional $\operatorname{Tr}(f(D / \Lambda))$, Eq. (8), accounts only for the bosonic term; the fermionic term can be included by adding $(1 / 2)\langle J \psi, D \psi\rangle$, where $J$ is the real structure on the spectral triple and $\psi$ is a spinor in the Hilbert space $\mathcal{H}$ of the quarks and leptons. The bosonic term is sufficient when cosmology is discussed; the fermionic part becomes essential when dealing with high energy consequences of the NCG spectral action.

It is important to emphasize that the NCG procedure outlined above is entirely classical, though it can a priori be quantized. The noncommutative spectral geometry simply provides an elegant way in which the SM of particle physics can be produced from purely (noncommutative) geometric information.

Another issue needs some attention. To use the formalism of spectral triples in NCG, it is convenient to work with Euclidean (i.e the signature is $(+,+,+,+)$ ) rather than Lorentzian signature. The discussion of phenomenological aspects of the theory relies on a Wick rotation to imaginary time ${ }^{6}$, into the standard (Lorentzian) signature. While sensible from the phenomenological point of view, there exists as yet no justification on the level of the underlying theory.

Applying the asymptotic expansion of Eq. (8) to the spectral action of the product geometry $\mathcal{M} \times \mathcal{F}$ gives a bosonic functional $S$ which includes cosmological terms, Riemannian curvature terms, Higgs minimal coupling, Higgs mass terms, Higgs quartic potential and Yang-Mills terms. The fact that the gravitational term includes, apart the Einstein-Hilbert action with a cosmological term and a topological term, also a conformal gravity

\footnotetext{
${ }^{6}$ In the Euclidean action functional for gravity, the kinetic terms must have the correct sign so that the functional is bounded below. Since such positivity is spoiled by the scalar Weyl mode, one must show that all other terms get a positive sign [2].
}

term with the Weyl curvature tensor and a conformal coupling of the Higgs field to gravity, essentially makes this model different from the usual minimal coupling of the SM to gravity. Another difference is that here the coefficients of the gravitational terms depend upon the Yukawa parameters of the particle physics content of the model.

In what follows, I will write explicitly the bosonic functional in Euclidean signature. One has to first perform a rescaling of the Higgs field $\varphi$ so that the kinetic terms are normalized. To normalize the Higgs field kinetic energy we rescale $\varphi$ to:

$$
\mathbf{H}=\frac{\sqrt{\mathfrak{a} f_{0}}}{\pi} \varphi,
$$

where the momentum $f_{0}$ is physically related to the coupling constants at unification and the coefficient $\mathfrak{a}$, related to the fermion and lepton masses and lepton mixing, is given by

$$
\begin{aligned}
\mathfrak{a}= & \operatorname{Tr}\left(Y_{(\uparrow 1)}^{\star} Y_{(\uparrow 1)}+Y_{(\downarrow 1)}^{\star} Y_{(\downarrow 1)}\right. \\
& \left.+3\left(Y_{(\uparrow 3)}^{\star} Y_{(\uparrow 3)}+Y_{(\downarrow 3)}^{\star} Y_{(\downarrow 3)}\right)\right),
\end{aligned}
$$

where the $(3 \times 3) Y$ matrices are used to classify the action of the Dirac operator and give the fermion and lepton masses, as well as lepton mixing, in the asymptotic version of the spectral action. The $Y$ matrices are only relevant for the coupling of the Higgs field with fermions through the dimensionless matrices $\pi / \sqrt{\mathfrak{a} f_{0}} Y_{x}$ with $x \in\{(\uparrow \downarrow, j)\}$. Thus, $\mathfrak{a}$ has the physical dimension of a (mass) $)^{2}$.

With this redefinition, the kinetic term reads

$$
\int \frac{1}{2}\left|D_{\mu} \mathbf{H}\right|^{2} \sqrt{g} d^{4} x
$$

The normalization of the kinetic terms imposes a relation between the coupling constants $g_{1}, g_{2}, g_{3}$ at unification and the coefficient $f_{0}$, namely

$$
\frac{g_{3}^{2} f_{0}}{2 \pi^{2}}=\frac{1}{4} \text { and } g_{3}^{2}=g_{2}^{2}=\frac{5}{3} g_{1}^{2}
$$

Performing an asymptotic expansion of the spectral action for the product geometry $\mathcal{M} \times \mathcal{F}$, the bosonic action in Euclidean signature reads [3]

$$
\begin{array}{r}
\mathcal{S}^{\mathrm{E}}=\int\left(\frac{1}{2 \kappa_{0}^{2}} R+\alpha_{0} C_{\mu \nu \rho \sigma} C^{\mu \nu \rho \sigma}+\gamma_{0}+\tau_{0} R^{\star} R^{\star}\right. \\
+\frac{1}{4} G_{\mu \nu}^{i} G^{\mu \nu i}+\frac{1}{4} F_{\mu \nu}^{\alpha} F^{\mu \nu \alpha}+\frac{1}{4} B^{\mu \nu} B_{\mu \nu} \\
+\frac{1}{2}\left|D_{\mu} \mathbf{H}\right|^{2}-\mu_{0}^{2}|\mathbf{H}|^{2} \\
\left.-\xi_{0} R|\mathbf{H}|^{2}+\lambda_{0}|\mathbf{H}|^{4}\right) \sqrt{g} d^{4} x
\end{array}
$$


where

$$
\begin{aligned}
\kappa_{0}^{2} & =\frac{12 \pi^{2}}{96 f_{2} \Lambda^{2}-f_{0} \mathfrak{c}}, \\
\alpha_{0} & =-\frac{3 f_{0}}{10 \pi^{2}} \\
\gamma_{0} & =\frac{1}{\pi^{2}}\left(48 f_{4} \Lambda^{4}-f_{2} \Lambda^{2} \mathfrak{c}+\frac{f_{0}}{4} \mathfrak{d}\right), \\
\tau_{0} & =\frac{11 f_{0}}{60 \pi^{2}}, \\
\mu_{0}^{2} & =2 \Lambda^{2} \frac{f_{2}}{f_{0}}-\frac{\mathfrak{e}}{\mathfrak{a}}, \\
\xi_{0} & =\frac{1}{12}, \\
\lambda_{0} & =\frac{\pi^{2} \mathfrak{b}}{2 f_{0} \mathfrak{a}^{2}},
\end{aligned}
$$

with $\mathfrak{a}$ given by Eq. (13) and $\mathfrak{b}, \mathfrak{c}, \mathfrak{d}, \mathfrak{e}$ given by 3

$$
\begin{aligned}
\mathfrak{b}= & \operatorname{Tr}\left(\left(Y_{(\uparrow 1)}^{\star} Y_{(\uparrow 1)}\right)^{2}+\left(Y_{(\downarrow 1)}^{\star} Y_{(\downarrow 1)}\right)^{2}\right. \\
& \left.+3\left(Y_{(\uparrow 3)}^{\star} Y_{(\uparrow 3)}\right)^{2}+3\left(Y_{(\downarrow 3)}^{\star} Y_{(\downarrow 3)}\right)^{2}\right), \\
\mathfrak{c}= & \operatorname{Tr}\left(Y_{R}^{\star} Y_{R}\right) \\
\mathfrak{d}= & \operatorname{Tr}\left(\left(Y_{R}^{\star} Y_{R}\right)^{2}\right), \\
\mathfrak{e}= & \operatorname{Tr}\left(Y_{R}^{\star} Y_{R} Y_{(\uparrow 1)}^{\star} Y_{(\uparrow 1)}\right),
\end{aligned}
$$

with $Y_{(\downarrow 1)}, Y_{(\uparrow 1)}, Y_{(\downarrow 3)}, Y_{(\uparrow 3)}$ and $Y_{R}$ being $(3 \times 3)$ matrices, with $Y_{R}$ symmetric.

Using Eq. (15) to replace $\sqrt{\mathfrak{a} f_{0}} / \pi$ by $\sqrt{\mathfrak{a}} /(g \sqrt{2})$, the notations of the Higgs fields change to [3]

$$
\begin{aligned}
\mathbf{H} & =\frac{\sqrt{\mathfrak{a}}}{g \sqrt{2}}(1+\psi), \\
& =\left(\frac{2 M}{g}+H-i \phi^{0}-i \sqrt{2} \phi^{+}\right),
\end{aligned}
$$

where $H, \phi^{0}, \phi^{+}$are Higgs fields and $M$ stands for the mass of the $W$ gauge boson.

The relations, Eq. (17), above rely on the validity of the asymptotic expansion at $\Lambda$, and are therefore tied intimately to the scale at which the expansion is performed. There is a priori no reason for the constraints to hold at scales below the unification scale $\Lambda$, since they represent mere boundary conditions.

The factor $f_{0}$ is fixed by the canonical normalization of the Yang-Mills terms (not included here) in terms of the common value of the gauge coupling constants $g$ at unification, $f_{0}=\pi^{2} /\left(2 g^{2}\right)$. The value of $g$ at unification scale is determined by standard renormalization group flow, i.e. it is given a value which reproduces the correct observed coupling at low energies, which is not unique since the gauge couplings fail to meet exactly in the nonsupersymmetric Standard Model (or its extension by right-handed neutrinos). The coefficients $\mathfrak{a}, \mathfrak{b}, \mathfrak{c}, \mathfrak{d}$ and $\mathfrak{e}$ are the Yukawa and Majorana parameters subject to renormalization group flow. Finally, the parameter $f_{2}$ is a priori unconstrained in $\mathbb{R}_{+}^{*}$.

Let me discuss the terms appearing in Eq. (16). The first two terms only depend upon the Riemann curvature tensor; the first is the Einstein-Hilbert term with the second one being the Weyl curvature term. Thus, the first two terms are the Riemannian curvature terms; the third one is the cosmological term. The fourth term

$$
R^{\star} R^{\star}=\frac{1}{4} \epsilon^{\mu \nu \rho \sigma} \epsilon_{\alpha \beta \gamma \delta} R_{\mu \nu}^{\alpha \beta} R_{\rho \sigma}^{\gamma \delta},
$$

is the topological term that integrates to the Euler characteristic, hence is nondynamical. The three next terms are the Yang-Mills terms. The eighth term is the scalar minimal coupling term, the next one is the scalar mass term, and the last one is the scalar quartic potential term. There is one more term, the $-\xi_{0} R|\mathbf{H}|^{2}$, that couples gravity with the SM. For $\xi_{0}=1 / 12$, this term encodes the conformal coupling between the Higgs field and the Ricci curvature. Let me sketch how this conformal coupling arises: The coupling term between the Higgs field and the Ricci curvature, appearing in the spectral action functional, is $-f_{0} /\left(12 \pi^{2}\right) \mathfrak{a} R|\phi|^{2}$, which after rescaling $\mathbf{H}=\left(\sqrt{\mathfrak{a} f_{0}} / \pi\right) \phi$, leads to the term $-R|\mathbf{H}|^{2} / 12$. This shows the conformal coupling ${ }^{7}$ between the background geometry and the Higgs field. Note that the coupling term between the Higgs field and the Ricci curvature should always be present when one considers gravity coupled to scalar fields. The nonminimal coupling between the Ricci curvature and the Higgs field, which appears naturally in noncommutative spectral geometry, can have significant consequences at high energies, such as in the early universe [13 18].

At this point, let me make a comment on the running of $\xi_{0}$, which will be useful later on, when I will discuss cosmological consequences of the NCG spectral action proposal. The constraint $\xi_{0}=1 / 12$ does not require by itself the coupling to remain conformal ${ }^{8}$, since it may run with the energy scale. Performing renormalization group analysis of the nonminimally coupled SM, it was argued [19, 20] that there are no quantum corrections to $\xi_{0}$, if it is exactly conformal at some energy scale. This claim was based on the observation that there are no nonconformal values for the coupling $\xi_{0}$ for which there is a renormalization group flow towards the conformal

\footnotetext{
7 Conformal invariance is considered here solely in the matter sector; the Einstein-Hilbert term is not conformally invariant.

8 This point is of a particular importance when one examines whether inflation through the Higgs field is a viable mechanism [14, 16].
} 
value as one runs the SM parameters up in the energy scale. Thus, if there is an exactly conformal coupling for the Higgs field at some specific scale, it will be exactly conformal at all scales.

In conclusion, the spectral action, Eq. (16), has to be considered as the bare action at unification scale $\Lambda$, where one supposes the merging of the coupling constants to take place. Since the NCG spectral action model lives naturally at unification scale, it provides a suitable setup to investigate early universe cosmological models. To make extrapolations to lower energy scales one has to use Renormalization Group Equations (RGE) ${ }^{9}$, and consider nonperturbative effects in the spectral action.

Assuming the big desert hypothesis, we can connect the physics at low energies with those at $E=\Lambda$ through the standard renormalization procedure. This was carried out at one-loop in Ref. [3], and more recently in Ref. [15] where Majorana mass terms for right-handed neutrinos were included and the see-saw mechanism was taken into account. Let me repeat that one has to be particularly careful, since the relations given in Eq. (17) cannot be taken as functions of the energy scale, this is simply incorrect; the relations in Eq. (17) hold only at unification scale $\Lambda$. This leads to a major difficulty when one would like to study lower energy astrophysical consequences of the noncommutative spectral geometry.

Furthermore, there is another complexity. Namely, it is very difficult to compute exactly the spectral action in its nonperturbative form, even though recently some progress has been achieved [12]. Since the action functional $\operatorname{Tr}(f(D / \Lambda))$ is not local, but its locality is only achieved when it is replaced by the asymptotic expansion, Eq. (9), one should at least compute the next term in the asymptotic expansion, in order to check its validity. It has been recently shown 12] that for a space-time whose spatial sections are 3 -spheres $S^{3}$, Wick rotated and compactified to a Euclidean model $S^{3} \times S^{1}$, the spectral action can be computed explicitly in a nonperturbative form, through the Poisson summation formula. For the $S^{3} \times S^{1}$, the authors of Ref. [12] have demonstrated that the spectral action is given, for any test function, by the sum of two terms up to a remarkably tiny correction; all higher order terms $a_{2 n}$ vanish. The authors have confirmed [12] their result by evaluating the spectral action using the heat kernel expansion and explicitly shown that both the higher order terms $a_{4}$ and $a_{6}$ vanish. Computations of the spectral action on other 3-manifolds, which however remain far of any realistic physical space, has been lately presented in Ref. 21].

\footnotetext{
9 The renormalized action will have the same form but with the bare quantities $\kappa_{0}, \alpha_{0}, \gamma_{0}, \tau_{0}, \mu_{0}, \xi_{0}, \lambda_{0}$, and the three gauge couplings $g_{1}, g_{2}, g_{3}$ replaced with physical quantities.
}

\section{PHENOMENOLOGICAL CONSEQUENCES OF THE NCG SPECTRAL ACTION}

Let us assume that the function $f$ is well approximated by the cut-off function, which then allows us to ignore higher order terms. The NCG spectral action approach then leads to the following phenomenological consequences [3]:

- Normalization of the kinetic terms dictates a relation between the coupling constants $g_{1}, g_{2}, g_{3}$ and the coefficient $f_{0}$, namely

$$
\frac{g_{3}^{2} f_{0}}{2 \pi^{2}}=\frac{1}{4} \text { and } g_{3}^{2}=g_{2}^{2}=\frac{5}{3} g_{1}^{2}
$$

(i.e., Eq. (15)).

- It consequently implies the relation

$$
\sin ^{2} \theta_{\mathrm{W}}=\frac{3}{8}
$$

which is also obtained in the context of the Grand Unified Theories (GUTs) SU(5) and $\mathrm{SO}(10)$.

- The three momenta $f_{0}, f_{2}, f_{4}$ can be used to specify the initial conditions on the gauge couplings, the Newton constant and the cosmological constant, as already discussed earlier.

- Assuming the big desert hypothesis, one can find the running of the three couplings $\alpha_{i}=g_{i}^{2} /(4 \pi)$. One-loop RGE for the running of the gauge couplings and the Newton constant, shows that they do not meet exactly at one point, the error is though within just few percent. Therefore, the model does not specify a unique unification energy. This negative result provides useful information about the nature of the function $f$ used in the spectral action. In the approach we have followed here, $f$ has been approximated by a cut-off function for which all coefficients, given by derivatives of $f$ at zero, of the higher order terms in the asymptotic expansion vanish. One can therefore conclude that the function $f$ can be safely approximated by the cut-off function, nevertheless there exist small deviations.

- There are 16 fundamental fermions.

- The correct representations of the fermions with respect to the gauge group, $\mathrm{G}_{\mathrm{SM}}$, of the $\mathrm{SM}$ are obtained.

- The Higgs doublet appears as part of the inner fluctuations of the metric, and Spontaneous Symmetry Breaking mechanism arises naturally with the negative mass term without any tuning. 
- The see-saw mechanism to give very light left-handed neutrinos is obtained.

Moreover, the model predicts [3]:

- A top quark mass of $M_{\text {top }} \sim 179 \mathrm{Gev}$.

- The mass of Higgs in zeroth order approximation of the spectral action is

$$
m_{H}=\sqrt{2 \lambda} \frac{2 M}{g} \sim 170 \mathrm{GeV},
$$

with $\lambda$ the quartic Higgs coupling. This value is however ruled out by current experimental data. Nevertheless, one should keep in mind that the result depends on the value of gauge couplings at unification scale, which is certainly uncertain. In addition, note that this result was found neglecting the nonminimal coupling between the Higgs field and the Ricci curvature.

Regarding the gravitational terms, neglecting (which is in principle incorrect) the nonminimal coupling between the Higgs field and the Ricci curvature, the noncommutative spectral geometry:

- Agrees with the very weak values of the coefficients of the quadratic curvature terms $R^{\mu \nu} R_{\mu \nu}$ and $R^{2}$ at low energies, found experimentally.

- For $\Lambda \sim 1.1 \times 10^{17} \mathrm{GeV}$, from the standard form of the gravitational action, $S(g)=1 /(16 \pi G) \int_{\mathrm{M}} R d v$, and the experimental value of Newton's constant at ordinary scales, finds the coupling constant to be

$$
\kappa_{0}\left(M_{Z}\right)=\sqrt{8 \pi G}
$$

thus,

$$
1 / \kappa_{0} \sim 2.43 \times 10^{18} \mathrm{GeV}
$$

Finally, the noncommutative geometry approach to unification does not provide any explanation of the number of generations, neither gives any constraints on the values of the Yukawa couplings.

I believe there are two, distinct and highly nontrivial, avenues along which the noncommutative geometry community has to make further progress. Firstly, to include higher order corrections to the spectral action and secondly, to find a noncommutative space, valid at unification scale, whose limit is the almost commutative geometry $\mathcal{M} \times \mathcal{F}$ we have considered insofar. Succeeding in these directions, may cure the small deviations found between the predictions of the SM derived from the noncommutative spectral geometry and the experimental values.

\section{COSMOLOGICAL CONSEQUENCES OF THE NCG SPECTRAL ACTION}

I will be using conventions in which the signature is $(-,+,+,+)$ and the Ricci tensor is defined ${ }^{10}$ as $R_{\mu \nu}=$ $R_{\mu \nu \rho}^{\rho}$, with $R_{\mu \nu \rho}{ }^{\sigma} \omega_{\sigma}=\left[\nabla_{\mu}, \nabla_{\nu}\right] \omega_{\rho}$.

The Lorentzian version of the gravitational part of the asymptotic formula for the bosonic sector of the NCG spectral action, including the coupling between the Higgs field and the Ricci curvature scalar, reads [3]

$$
\begin{array}{r}
\mathcal{S}_{\text {grav }}^{\mathrm{L}}=\int\left(\frac{1}{2 \kappa_{0}^{2}} R+\alpha_{0} C_{\mu \nu \rho \sigma} C^{\mu \nu \rho \sigma}+\tau_{0} R^{\star} R^{\star}\right. \\
\left.-\xi_{0} R|\mathbf{H}|^{2}\right) \sqrt{-g} d^{4} x .
\end{array}
$$

The equations of motion arising from Eq. (24) read [13]

$$
\begin{array}{r}
R^{\mu \nu}-\frac{1}{2} g^{\mu \nu} R+\frac{1}{\beta^{2}} \delta_{\mathrm{cc}}\left[2 C_{; \lambda ; \kappa}^{\mu \lambda \nu \kappa}+C^{\mu \lambda \nu \kappa} R_{\lambda \kappa}\right] \\
=\kappa_{0}^{2} \delta_{\mathrm{cc}} T_{\text {matter }}^{\mu \nu}
\end{array}
$$

where $\beta^{2}$ and $\delta_{\text {cc }}$ are defined as

$$
\beta^{2} \equiv-\frac{1}{4 \kappa_{0}^{2} \alpha_{0}},
$$

and

$$
\delta_{\mathrm{cc}} \equiv\left[1-2 \kappa_{0}^{2} \xi_{0} \mathbf{H}^{2}\right]^{-1}
$$

respectively.

\section{Low energy regime}

Let me first concentrate on the low energy weak curvature regime, where the nonminimal coupling term between the background geometry and the Higgs field is small and can be safely neglected. This implies that $\delta_{\mathrm{cc}}=1$. For a Friedmann-Lemaître-RobertsonWalker (FLRW) space-time, the Weyl tensor vanishes, hence the NCG corrections to the Einstein equation vanish [13]. This result has important consequences. Since the NCG corrections vanish for FLRW cosmologies and Schwarzschild solutions, it is difficult to place restrictions on $\beta^{2}$ (or equivalently on $\alpha_{0}$, or on $f_{0}$ ), via cosmology or solar-system tests. Note that the best constraint on, different ad hoc, curvature squared terms is obtained from measurements of the orbital precession of Mercury, imposing the rather weak lower bound [22] $\beta_{R^{2}} \geq 3.2 \times 10^{-9} \mathrm{~m}^{-1}$. Since this constraint was found

10 In General Relativity such choices are merely conventions, which are relatively unimportant; in the NCG approach we are following here the situation is however very different. 
for terms of different form (but of the same order) to the Weyl term appearing in the NCG spectral action, it does not necessarily hold here.

We have constrained $\beta$ within the NCG spectral action context in Ref. [18]. This constraint is very important, since by imposing a lower limit to $\beta$, we actually set an upper limit to the moment $f_{0}$ of the cut-off function used to define the spectral action. Since $f_{0}$ can be used to specify the initial conditions on the gauge couplings (see Eq. (15)), a constraint on $\beta$ corresponds to a restriction on the particle physics at unification. I will briefly summarize how this constraint has been obtained [17, 18].

Consider linear perturbations around a Minkowski background metric in the synchronous gauge. The perturbed metric reads

$$
g_{\mu \nu}=\operatorname{diag}\left(\{a(t)\}^{2}\left[-1,\left(\delta_{i j}+h_{i j}(x)\right)\right]\right),
$$

where $a(t)$ is the cosmological scale factor. Since we only consider a flat background, $a(t)=1$ and $\dot{a} \equiv d a / d t=0$. Note that the remaining gauge freedom can be completely fixed by setting $\nabla_{i} h^{i j}=0$.

The linearized equations of motion derived from the NCG spectral action for such perturbations read [17]

$$
\left(\square-\beta^{2}\right) \square h^{\mu \nu}=\beta^{2} \frac{16 \pi G}{c^{4}} T_{\text {matter }}^{\mu \nu},
$$

where $T_{\text {matter }}^{\mu \nu}$ is taken to lowest order in $h^{\mu \nu}$. This implies that it is independent of $h^{\mu \nu}$ and satisfies the conservation equations

$$
\frac{\partial}{\partial x^{\mu}} T_{\nu}^{\mu}=0
$$

Note that $\beta$ plays the rôle of a mass and hence has to be real and positive, thus $\alpha_{0}<0$. For $\alpha_{0}>0$, as it has been explicitly shown in Ref. [17], the gravitational waves evolve according to a Klein-Gordon like equation with a tachyonic mass, and hence the background, which has been considered to be a Minkowski space, is unstable. One hence has to restrict to $\alpha_{0}<0$ for Minkowski space to be a (stable) vacuum of the theory.

Let us study the energy lost to gravitational radiation by orbiting binaries. In the far field limit, $|\mathbf{r}| \approx\left|\mathbf{r}-\mathbf{r}^{\prime}\right|$ (where $\mathbf{r}$ and $\mathbf{r}^{\prime}$ stand for the locations of the observer and emitter, respectively), the spatial components of the general first order solution for a perturbation against a Minkowski background read [17]

$$
\begin{aligned}
& h^{i k}(\mathbf{r}, t) \approx \frac{2 G \beta}{3 c^{4}} \int_{-\infty}^{t-\frac{1}{c}|\mathbf{r}|} \frac{d t^{\prime}}{\sqrt{c^{2}\left(t-t^{\prime}\right)^{2}-|\mathbf{r}|^{2}}} \\
& \times \mathcal{J}_{1}\left(\beta \sqrt{c^{2}\left(t-t^{\prime}\right)^{2}-|\mathbf{r}|^{2}}\right) \ddot{D}^{i k}\left(t^{\prime}\right),
\end{aligned}
$$

with $\mathcal{J}_{1}$ is a Bessel function of the first kind, in terms of the quadrupole moment,

$$
D^{i k}(t) \equiv \frac{3}{c^{2}} \int x^{i} x^{k} T^{00}(\mathbf{r}, t) d \mathbf{r} .
$$

As one can easily check from Eq. (25), the theory reduces to that of General Relativity in the $\beta \rightarrow \infty$ limit and one can reproduce the familiar result for a massless graviton. For finite $\beta$ however, gravitational radiation contains both massive and massless modes, both of which are sourced from the quadrupole moment of the system.

Consider a binary pair of masses $m_{1}, m_{2}$ in a circular (for simplicity) orbit in the (xy)-plane. The rate of energy loss from such a system, in the far field limit, is

$$
-\frac{\mathrm{d} \mathcal{E}}{\mathrm{d} t} \approx \frac{c^{2}}{20 G}|\mathbf{r}|^{2} \dot{h}_{i j} \dot{h}^{i j},
$$

with [17]

$$
\begin{aligned}
\dot{h}^{i j} \dot{h}_{i j} & =\frac{128 \mu^{2}|\rho|^{4} \omega^{6} G^{2} \beta^{2}}{c^{8}} \\
& \times\left[f_{\mathrm{c}}^{2}\left(\beta|\mathbf{r}|, \frac{2 \omega}{\beta c}\right)+f_{\mathrm{s}}^{2}\left(\beta|\mathbf{r}|, \frac{2 \omega}{\beta c}\right)\right],
\end{aligned}
$$

where we have defined the functions:

$$
\begin{aligned}
& f_{\mathrm{s}}(x, z) \equiv \int_{0}^{\infty} \frac{d s}{\sqrt{s^{2}+x^{2}}} \mathcal{J}_{1}(s) \sin \left(z \sqrt{s^{2}+x^{2}}\right), \\
& f_{\mathrm{c}}(x, z) \equiv \int_{0}^{\infty} \frac{d s}{\sqrt{s^{2}+x^{2}}} \mathcal{J}_{1}(s) \cos \left(z \sqrt{s^{2}+x^{2}}\right) ;
\end{aligned}
$$

$\omega$ stands for the orbital frequency, which for the system under consideration is a constant given by

$$
\omega=|\rho|^{-3 / 2} \sqrt{G\left(m_{1}+m_{2}\right)},
$$

with $|\rho|$ the magnitude of the separation vector between the two bodies.

The integrals in Eq. (35), which can be easily evaluated for $z<1$ and $z>1$, exhibit a strong resonance behavior at $z=1$, which corresponds to the critical frequency [17]

$$
2 \omega_{\mathrm{c}}=\beta c,
$$

around which strong deviations from the familiar results of General Relativity are expected. This critical (maximum) frequency comes from the natural length scale (given by $\beta^{-1}$ ) in the NCG theory, at which noncommutative geometry effects become dominant.

For $z<1$ and $z>1$, the functions in Eq. (35) can be evaluated numerically and fitted to an explicit functional form [17]. It can be then easily checked that for $\omega<$ $\omega_{\mathrm{c}}$, the $\beta \rightarrow \infty$ limit reproduces the General Relativity result, as it should. Since this is not the case for the $\omega>\omega_{\mathrm{c}}$ case, we consider the critical frequency as the maximum one. Any deviation from the standard result is suppressed by the distance to the source, at least for 
orbital frequencies small compared to $\beta c$.

For the physically interesting case of $\omega<\omega_{\mathrm{c}}$, even though the amplitude of the deviation from the standard result is small, due to the $1 /|\mathbf{r}|$ suppression, one should notice the existence of a critical frequency $\omega_{c}$ and the oscillatory nature of the rate of flux of gravitational radiation with changing distances and changing frequencies.

The form of the gravitational radiation from binary systems can be now used to constrain $\beta$. Since we have considered only circular binary orbits, we only need to know the orbital frequency and the distance to the binary system. Several binary pulsars, for which the rate of change of the orbital frequency has been well characterized, and the predictions of General Relativity agree with the data to high accuracy have been used in Ref. [18]. The parameter $\beta$ has been then restricted by requiring that the magnitude of deviations from General Relativity be less than this uncertainty. Using these data and requiring that $\omega<\omega_{\mathrm{c}}$, we found [18]

$$
\beta>7.55 \times 10^{-13} \mathrm{~m}^{-1} .
$$

Even though this observational constraint may seem weak, it is comparable to (but larger than) existing constraints on similar, ad hoc, additions to General Relativity, and one expects that it will rapidly be improved as more binary pulsars are discovered and the observations of existing systems improve.

Due to the large distances to these binary systems, this constraint, Eq. (37), is almost exactly due to $\beta>2 \omega / c$. Thus, the strongest constraint comes from systems with high orbital frequencies. Future observations of rapidly orbiting binaries, relatively close to the Earth, could thus improve this constraint by many orders of magnitude. Nevertheless, it is indeed remarkable that the value of the Weyl squared coupling in the bosonic action could be constrained [18] via astrophysical data.

Let me go back to the background equations. In order for the corrections to Einstein's equations to be apparent at leading order, i.e. at the level of the background, one needs to consider anisotropic models. As an example, calculate the modified Friedmann equation for the Bianchi type- $\mathrm{V}$ model, for which the space-time metric in Cartesian coordinates reads

$$
g_{\mu \nu}=\operatorname{diag}\left[-1,\left\{a_{1}(t)\right\}^{2} e^{-2 n z},\left\{a_{2}(t)\right\}^{2} e^{-2 n z},\left\{a_{3}(t)\right\}^{2}\right],
$$

where $a(t), b(t)$ and $c(t)$ are, in general, arbitrary functions and $n$ is an integer.

Writing down the modified Friedmann equation, we have found [13] that the correction terms come in two types. The first one contains terms which are fourth order in time derivatives. Hence for the slowly varying functions, usually used in cosmology, they can be taken to be small corrections. The second one occurs at the same order as the standard Einstein-Hilbert terms. However, it is proportional to $n^{2}$ and hence vanishes for homogeneous versions of Bianchi type- $\mathrm{V}$. Thus, although anisotropic cosmologies do contain corrections due to the additional NCG terms in the action, they are typically of higher order. Inhomogeneous models do contain correction terms that appear on the same footing as the original (commutative) terms. In conclusion, the corrections to Einstein's equations are present only in inhomogeneous and anisotropic space-times.

\section{High energy regime}

At energies approaching the Higgs scale, the nonminimal coupling of the Higgs field to the curvature can no longer be neglected, leading to corrections even for background cosmologies. To understand the effects of these corrections let us neglect the conformal term in Eq. (25), i.e. $\operatorname{set} \beta=0$. The equations of motion then become 13]

$$
R^{\mu \nu}-\frac{1}{2} g^{\mu \nu} R=\kappa_{0}^{2}\left[\frac{1}{1-\kappa_{0}^{2}|\mathbf{H}|^{2} / 6}\right] T_{\text {matter }}^{\mu \nu} .
$$

Hence, the $|\mathbf{H}|$ leads to an effective gravitational constant.

Alternatively, consider the effect of this term on the equations of motion for the Higgs field in a constant gravitational field. For constant curvature, the self interaction of the Higgs field is increased, as one can easily see from [13],

$$
-\mu_{0}|\mathbf{H}|^{2} \rightarrow-\left(\mu_{0}+\frac{R}{12}\right)|\mathbf{H}|^{2} .
$$

The nonminimal coupling between the Higgs field and the Ricci curvature may turn out to be particularly useful in early universe cosmology [14, 16]. Such a coupling has been introduced ad hoc in the literature, in an attempt to drive inflation through the Higgs field. However, the coupling constant between the scalar field and the background geometry is not a free parameter which could be tuned to achieve a successful inflationary scenario, it should be instead dictated by the underlying theory.

Let me write again the Gravity-Higgs sector of the asymptotic expansion of the spectral action, in 
Lorentzian signature $^{11}$,

$$
\begin{array}{r}
S_{\mathrm{GH}}^{\mathrm{L}}=\int\left[\frac{1-2 \kappa_{0}^{2} \xi_{0} H^{2}}{2 \kappa_{0}^{2}} R\right. \\
\left.-\frac{1}{2}(\nabla H)^{2}-V(H)\right] \sqrt{-g} d^{4} x,
\end{array}
$$

where

$$
V(H)=\lambda_{0} H^{4}-\mu_{0}^{2} H^{2},
$$

with $\mu_{0}$ and $\lambda_{0}$ subject to radiative corrections as functions of energy. For large enough values of the Higgs field, the renormalized value of these parameters must be calculated, while the running of the top Yukawa coupling and the gauge couplings must be evolved simultaneously.

At high energies the mass term is sub-dominant, and can be neglected (only the first term in Eq. (42) survives). For each value of the top quark mass, there is a value of the Higgs mass where the effective potential is on the verge of developing a metastable minimum at large values of the Higgs field and the Higgs potential is locally flattened [16]. Note that since the region where the potential becomes flat is narrow, slow-roll must be very slow, in order to provide a sufficiently long period of quasi-exponential expansion, necessary to solve the shortcomings of the standard Hot Big Bang cosmological model. Besides the slow-roll parameters, denoted by $\epsilon$ and $\eta$, which may be slow enough to get sufficient e-folds, the amplitude of density perturbations $\Delta_{\mathcal{R}}^{2}$ in the Cosmic Microwave Background must be in agreement with the measured one. Inflation predicts that at horizon crossing (denoted by stars), the amplitude of density perturbations is related to the inflaton potential through

$$
\left(\frac{V_{*}}{\epsilon_{*}}\right)^{\frac{1}{4}}=2 \sqrt{3 \pi} m_{\mathrm{Pl}} \Delta_{\mathcal{R}}^{\frac{1}{2}},
$$

where $\epsilon_{*} \leq 1$. Its value, as measured by WMAP7 23], requires

$$
\left(\frac{V_{*}}{\epsilon_{*}}\right)^{\frac{1}{4}}=(2.75 \pm 0.30) \times 10^{-2} m_{\mathrm{Pl}}
$$

where $m_{\mathrm{Pl}}$ stands for the Planck mass.

In Ref. [16] we have calculated the renormalization of the Higgs self-coupling up to two-loops and then constructed an effective potential which fits the renormalization group improved potential around the flat region. We have found [16] that around the plateau (the minimum of the potential), there is a very good analytic fit

11 Since we are dealing with a FLRW metric, the Weyl tensor vanishes; the nondynamical term is also neglected. to the Higgs potential, which takes the form

$$
\begin{aligned}
V^{\mathrm{eff}} & =\lambda_{0}^{\mathrm{eff}}(H) H^{4} \\
& =\left[a \ln ^{2}(b \kappa H)+c\right] H^{4},
\end{aligned}
$$

where the parameters $a, b$ are found to relate to the low energy values of top quark mass $m_{\mathrm{t}}$ as [16]

$$
\begin{aligned}
a\left(m_{\mathrm{t}}\right)= & 4.04704 \times 10^{-3}-4.41909 \times 10^{-5}\left(\frac{m_{\mathrm{t}}}{\mathrm{GeV}}\right) \\
& +1.24732 \times 10^{-7}\left(\frac{m_{\mathrm{t}}}{\mathrm{GeV}}\right)^{2}, \\
b\left(m_{\mathrm{t}}\right)= & \exp \left[-0.979261\left(\frac{m_{\mathrm{t}}}{\mathrm{GeV}}-172.051\right)\right] .
\end{aligned}
$$

The third parameter, $c=c\left(m_{\mathrm{t}}, m_{\phi}\right)$, encodes the appearance of an extremum and depends on the values for top quark mass and Higgs mass. An extremum occurs if and only if $c / a \leq 1 / 16$, the saturation of the bound corresponding to a perfectly flat region. It is convenient to write $c=[(1+\delta) / 16] a$, where $\delta=0$ saturates the bound below which a local minimum is formed.

Note that the above strictly holds for the case of minimal coupling, whereas in NCG we have a small nonminimal coupling, $\xi_{0}=1 / 12$. The corrections due to conformal coupling to the potential imply that flatness does not occur at $\delta=0$ anymore but for fixed values of $\delta$ depending on the value of the top quark mass. More precisely, for inflation to occur via this mechanism, the top quark mass fixes the Higgs mass extremely accurately. Scanning through parameter space it emerges that sufficient $e$-folds are indeed generated provided a suitably tuned relationship between the top quark mass and the Higgs mass holds [16].

The exhaustive study of Ref. [16] has shown that while the Higgs potential can lead to the slow-roll conditions being satisfied once the running of the self-coupling at two-loops is included, the constraints imposed from the CMB data make the predictions of such a scenario incompatible with the measured value of the top quark mass.

Finally, running of the gravitational constant and corrections by considering the more appropriate de Sitter, instead of a Minkowski, background do not improve substantially the realization of a successful inflationary era [16].

The NCG Spectral Action provides in addition to the Higgs field, another (massless) scalar field [24], denoted by $\sigma$, which is unlike all other fields in the theory, such as the Higgs field and gauge fields. Note that $\sigma$ does not exhibit a coupling to the matter sector.

Including this field, the cosmologically relevant terms 
in the Wick rotated action read 24]

$$
\begin{array}{r}
S=\int\left[\frac{1}{2 \kappa^{2}} R-\xi_{H} R H^{2}-\xi_{\sigma} R \sigma^{2}\right. \\
\left.-\frac{1}{2}(\nabla H)^{2}-\frac{1}{2}(\nabla \sigma)^{2}-V(H, \sigma)\right] \sqrt{-g} d^{4} x,
\end{array}
$$

where

$$
V(H, \sigma)=\lambda_{H} H^{4}-\mu_{H}^{2} H^{2}+\lambda_{\sigma} \sigma^{4}+\lambda_{H \sigma}|H|^{2} \sigma^{2} .
$$

The constants are related to the underlying parameters as follows:

$$
\begin{aligned}
& \xi_{H}=\frac{1}{12} \quad, \quad \xi_{\sigma}=\frac{1}{12} \\
& \lambda_{H}=\frac{\pi^{2} \mathfrak{b}}{2 f_{0} \mathfrak{a}^{2}} \quad, \quad \lambda_{\sigma}=\frac{\pi^{2} \mathfrak{d}}{f_{0} \mathfrak{c}^{2}} \\
& \mu_{H}=2 \Lambda^{2} \frac{f_{2}}{f_{0}} \quad, \quad \lambda_{H \sigma}=\frac{2 \pi^{2} \mathfrak{e}}{a \mathfrak{c} f_{0}} .
\end{aligned}
$$

Unfortunately, neither the $\sigma$ field can lead to a successful slow-roll inflationary era, if the coupling values are conformal [16].

\section{CONCLUSIONS}

Noncommutative spectral geometry is a beautiful mathematical construction which offers an elegant and purely geometric interpretation of the Standard Model of electroweak and strong interactions.

According to this proposal, the geometry near the Planck energy scale is the tensor product of an internal discrete geometry for the SM and a continuous geometry for space-time. The unification is based on the symplectic unitary group in Hilbert space and on the spectral action.

The NCG approach yields all the detailed structure of the SM with a very little input, while it provides predictions at unification scale. In particular, besides the familiar predictions for the gauge couplings in agreement with GUTs theories, the model predicts the Higgs scattering parameter and the sum of the squares of Yukawa couplings.

Since the model lives by construction at unification scale, it provides an excellent framework to address early universe cosmological questions, while astrophysical is- sues are more difficult to be dealt.

In this contribution, I have first summarized the main mathematical elements of noncommutative spectral geometry and then discussed briefly some of its phenomenological and cosmological consequences.

[1] A. Connes, Noncommutative Geometry, Academic Press, New York (1994).

[2] A. Connes and M. Marcolli, Noncommutative Geometry, Quantum Fields and Motives, Hindustan Book Agency, India (2008).

[3] A. H. Chamseddine, A. Connes and M. Marcolli, Adv. Theor. Math. Phys. 11, 991 (2007) arXiv:hep-th/0610241.

[4] A. Connes, JHEP 0611,081 arXiv:hep-th/0608226.

[5] J. Barrett, J. Math. Phys. 48, 012303 (2007).

[6] A. H. Chamseddine, A. Connes, J. Geom. Phys. 58, 38 (2008) arXiv:0706.3688 [hep-th]].

[7] A. H. Chamseddine and A. Connes, Phys. Rev. Lett. 99, 191601 (2007) arXiv:0706.3690 [hep-th]].

[8] A. H. Chamseddine and A. Connes, J. of Math. Phys. 47, 063504 (2006) arXiv:hep-th/0512169.

[9] A. H. Chamseddine and A. Connes, J. Math. Phys. 47, 063504 (2006).

[10] A. H. Chamseddine and A. Connes, Phys. Rev. Lett. 77, 4868 (1996).

[11] A. H. Chamseddine and A. Connes, Comm. Math. Phys. 186, 731 (1977).

[12] A. H. Chamseddine and A. Connes, Comm. Math. Phys. 293, 867 (2010) arXiv:0812.0165 [hep-th].

[13] W. Nelson and M. Sakellariadou, Phys. Rev. D 81, 085038 (2010) arXiv:0812.1657 [hep-th]].

[14] W. Nelson and M. Sakellariadou, Phys. Lett. B 680, 263 (2009) arXiv:0903.1520 [hep-th]].

[15] M. Marcolli and E. Pierpaoli, arXiv:0908.3683 [hep-th].

[16] M. Buck, M. Fairbairn and M. Sakellariadou, Phys. Rev. D 82, 043509 (2010) arXiv:1005.1188 [hep-th]].

[17] W. Nelson, J. Ochoa and M. Sakellariadou, arXiv:1005.4276 [hep-th]].

[18] W. Nelson, J. Ochoa and M. Sakellariadou, arXiv:1005.4279 [hep-th]].

[19] I. L. Buchbinder, S. D. Odintsov and I. M. Lichtzier, Class. Quant. Grav. 6, 605 (1989).

[20] Youngsoo Yoon and Yongsung Yoon, Int. J. Mod. Phys. A 12, 2903 (1997.

[21] M. Marcolli E. Pierpaoli and K. Teh, arXiv:1005.2256 [hep-th]].

[22] K. S. Stelle, Gen. Rel. Grav. 9353 (1978).

[23] D. Larson et al., arXiv:1001.4758 [astro-ph]].

[24] A. H. Chamseddine, arXiv:0901.0577 [hep-th]]. 